\title{
Mapping quantitative trait loci (QTLs) associated with dough quality in a soft $\times$ hard bread wheat progeny
}

\author{
Samir Kerfal ${ }^{a, 1}$, Patricia Giraldo ${ }^{a}$, Marta Rodríguez-Quijano ${ }^{a}$, J. Francisco Vázquez ${ }^{a}$, Kathy Adams ${ }^{b}$, \\ Odean M. Lukow ${ }^{\mathrm{b}}$, Marion S. Röder ${ }^{\mathrm{c}}$, Daryl J. Somers ${ }^{\mathrm{d}}$, José M. Carrillo ${ }^{\mathrm{a}, *}$ \\ anidad de Genética, Departamento de Biotecnología, E.T.S.IA., Universidad Politécnica de Madrid, Ciudad Universitaria s/n, 28040 Madrid, Spain \\ ${ }^{b}$ Agriculture and Agri-Fond Canada, Cereal Research Centre, 195 Dafoe Rd., Winnipeg, MB R3T 2M9, Canada \\ "Institut für Pfanzengenetik und Kulturpflanzenforschung (IPK), 06466 Gatersleben, Germany \\ dVineland Research and innovation Centre, 4890 Victoria Ave. North, Lincoin, ON LOR 2E0, Canada
}

Keywords:

Recombinant inbred lines

Storage proteins

Quantitative trait loci

Bread wheat quality

\begin{abstract}
A B S T R A C T
Bread wheat (Triticum aestivum L.) quality is a key trait for baking industry exigencies and broad consumer preferences. The main goal of this study was to undertake quantitative trait loci (QTL) analyses for bread wheat quality in a set of 79 recombinant inbred lines (RILS) derived from a soft $\times$ hard bread wheat cross. Field trials were conducted over two years, utilizing a randomized complete block design Dough quality was evaluated by sedimentation test, mixograph and alveograph analysis. Protein content was measured by near-infrared reflectance analysis and grain hardness was determined by the single kernel characterization system (SKCS).

A genetic map based on 263 SSR markers and glutenin loci was constructed. Composite interval mapping (CIM) analysis detected a total of 20 QTLs distributed among ten chromosomes which were associated with variations in quality traits.

Results confirmed the previous investigations on the known relationship between storage-protein alleles and dough quality, and detected new and stable QTLs related to dough quality parameters on chromosomes 2A, 7A, 5B and 1D. These new QTLs could be further investigated. Also, in this study, some RILs showed very high dough extensibility values which involve future validation studies for QTLs associated with to this trait.
\end{abstract}

\section{Introduction}

Wheat (Triticum aestivum L.) breeding programs have mainly focused on creating new varieties with high grain yield and resistance to abiotic and bjotic stress. However, baking industry exigencies and wide consumer preferences have driven wheat breeders to incorporate the trait 'wheat quality' as an important target goal in their current research (Bushuk, 1998).

End-use quality is defined as the suitability of wheat flour for producing specific end-products such as: bread, pastry, cakes, noodles, breakfast cereals or crackers. It depends on wheat, flour and dough properties. It is mainly determined by the quantity and quality of gluten proteins, key components of the endosperm (Finney, 1943; Payne et al., 1987). Strong wheat is considered to have good bread making quality, while weak wheat is considered to have poor bread making quality (Pomeranz, 1988).

Rheological theory related to cereals was used to describe the main processes presented during bread making (mixing, sheeting, fermentation and baking). The most commonly used rheological test methods and their relationships to product functionality were reviewed by Dobraszczyk and Morgenstern (2003). For example, dough mixing could be predicted by $10 \mathrm{~g}$ mixograph, dough uniaxial extensibility by extensigraph and dough biaxial extensibility by alveograph.

Gluten proteins are composed of glutenins and gliadins. Glutenins are divided into two groups: high molecular weight glutenin 
subunits (HMW-GS) and low molecular weight glutenin subunits (LMW-GS) (Payne and Corfield, 1979). Gliadins were classified into four groups on the basis of mobility at low $\mathrm{pH}$ in gel electrophoresis. Several studies have reported the relationship between HMW-GS, LMW-GS, gliadins and dough quality. In general, the gliadins are associated with extensibility of the dough, while the glutenins are involved in strength and elasticity (MacRitchie, 1994).

In fact, dough quality traits are generally under multi-gene control and cannot be fully explained by loci coding for storage proteins. These traits are frequently influenced by environmental factors and show evidence of high genotype $\times$ environment interaction. A better understanding of this genetic control would enhance the development of superior bread wheat cultivars with high dough quality.

Current researches in molecular marker technologies and quantitative trait analysis have permitted scientists to identify and estimate the effects of quantitative trait loci (QTLs) associated with quality traits. In wheat, QTLs have been mapped for many quality traits such as grain protein content (Blanco et al., 2002; Campbell et al., 2001; Groos et al., 2003; Joppa et al., 1997; Sourdille et al.. 2003; Suprayogui et al., 2009; Turner et al., 2004; Zanetti et al., 2001), grain hardness (Sourdille et al., 2003; Turner et al., 2004; Zanetti et al., 2001), and dough quality traits such as mixing time, mixing tolerance, dough tenacity and dough extensibility (Campbell et al., 2001; Cornish et al., 2001; Crepieux et al., 2005; Elangovan et al., 2008; Huang et al., 2006; Li et al., 2009; Ma et al., 2007; Mann et al., 2009; Nelson et al., 2006; Patil et al., 2009; Perretant et al., 2000; Sourdille et al., 2003; Zanetti et al., 2001).

We have analyzed a segregated population derived from a cross between 'Marius', a French soft-grained variety with high dough extensibility, and 'Cajeme71', a hard-grained variety from CYMMIT with high dough gluten strength. Within this population, grown in two seasons, we have searched for novel QTLs associated with gluten strength and extensibility, the main factors of dough quality. These two traits have been measured by sedimentation test, mixograph and alveograph parameters, the main techniques used for estimating bread quality.

\section{Materials and methods}

\subsection{Plant material and experimental design}

The population used in this work consisted of $79 \mathrm{~F}_{7}$ recombinant inbred lines (RILs) obtained by single seed descent from a cross between two cultivars: 'Marius' a French winter wheat, softgrained with a high dough extensibility and 'Cajeme71' a spring wheat (developed in Mexico by N. E. Borlaug in 1960s, CIMMYT), hard-grained with a higher gluten strength. Field trials were conducted over two years (harvest years 2006 and 2007) utilizing a randomized complete block design with 2 replicates at the experimental field of the Escuela Técnica Superior Ingenieros Agrónomos, Universidad Politécnica de Madrid, Spain $\left(40^{\circ} 26^{\prime} 47.36^{\prime \prime} \mathrm{N}, 3^{\circ} 44^{\prime} 21.00^{\prime \prime} \mathrm{W}\right)$.

\subsection{Electrophoresis and nomenclature}

Allelic variation of HMW-GS and LMW-GS from the RILs was determined using sodium dodecyl sulfate polyacrylamide gel electrophoresis (SDS-PAGE) as previously described (Payne, 1987). The LMW-GS were named following the method described by Gupta and Shepherd (1990). The gliadin banding patterns were separated by acid polyacrylamide gel electrophoresis (A-PAGE) (Lafiandra and Kasarda, 1985) and numbered according to established nomenclature (Metakovsky et al., 2000).

\subsection{Genetic map construction and QTL analysis}

Extraction of genomic DNA, PCR amplification, PCR screening and genotyping data was performed as previously described by Somers et al. (2004), using M13 tailing and fluorescent capillary electrophoresis on an ABI 3100 Genetic Analyzer (Applied Biosystems, Foster City, CA, USA).

Six hundred and eighty-three SSR markers were analyzed in the parental lines while linkage analysis was based only on a total of 263 polymorphic microsatellite markers (GWM, GDM, CFD, WMC, and BARC) and eight genes/gene loci (Glu-A1, Ghu-B1, Glu-D1, Glu$A 3, G h-B 3, G h-D 3, G(i-A 2$ and $G(i-B 2)$ distributed along the 21 wheat chromosomes. The genetic map was constructed with MAPMAKER/ Exp version 3.0b (Lander et al.., 1987). The commands "group" with LOD scores equal a 3.0, "try", "compare" and "ripple" were used to develop the linkage map. Unlinked groups were oriented and placed to the same chromosome based on the microsatellite consensus map (Somers et al., 2004). The Kosambi mapping function was used to convert recombination fractions into centiMorgans (cM) as map distances (Kosambi, 1944).

QTL analysis was performed by CIM analysis and carried out with QTL-Cartographer 1.3 (Basten et al., 2001). A window size of $10 \mathrm{cM}$ around the test interval was chosen in all analysis. The threshold LOD scores for detection of significant QTLs were calculated by 1000 permutation test, LOD thresholds of 2.5 were chosen for CIM. The proportion of phenotypic variance explained by each QTL marker was estimated using the coefficient of determination $\left(R^{2}\right)$.

\subsection{Quality analysis}

Protein concentration (PC) ( $14 \%$ moisture basis) was measured by near-infrared reflectance analysis using the Technichon Infranalyzer 300 . Hardness index (HI) ("hard" or "soft" kernel texture) was determined on an approximately 300-kernel subsample by the Perten Model SKCS 4100 (Perten lnstiuments North America Inc., Springfield, IL). Sodium dodecyl sulfate sedimentation test (SDSS) was performed using $1 \mathrm{~g}$ flour of each sample (Dick and Quick, 1983). Mixograph analysis was carried out using a $10 \mathrm{~g}$ mixograph system (Mixograph National Manufacturing $\mathrm{CO}$, Lincoln, NE, USA) and the AACC 54-40A method (AACC Approved Methods, 1995). The mixograph parameters measured were: mixing development time (MxT), maximum peak height $(\mathrm{MH})$, and mixing tolerance (MTo, difference in percentage between $\mathrm{MH}$ and height at 3 min after the peak of the curve).

The rheological properties of the dough prepared from wheat flour were carried out on a Chopin MA 82 Alveograph (Tripette \& Renaud, Villeneuve-la-Garenne, France). Dough characteristics such as dough tenacity (DTen), dough extensibility (DExt) and dough strength (DStren) were recorded.

Statistical analysis of phenotypic data was carried out using PROC MIXED of SAS (SAS Institute lnc., 1996) where block were considered as random effects and genotype and environment (two years) as fixed effects. Transgressive segregation among RlLs, Marius, and Cajeme71 was tested by the Tukey-Kramer test at $P<0.05$. A macro (Pdmix 800) was used for converting mean separation output to letter groupings in Proc Mixed (Saxton, 1998).

\section{Results and discussion}

\subsection{Biochemical and phemotypic variation of parental lines and RILs' population}

The HMW-GS, LMW-GS and gliadin alleles detected in the parental lines are presented in Table 1 . A total of nine different HMW-GS protein bands coded by three genetic loci Glu-A1, Glu-B1 
Table 1

Subunitsfalleles detected in the parental lines (Marius and Cajeme71) at glutenin and gliadin toci.

\begin{tabular}{|c|c|c|}
\hline & Marius & Cajeme71 \\
\hline$G(u-A)$ & Null/c & $1 / a$ \\
\hline Giu-BI & $7+9 / c$ & $17+18 f i$ \\
\hline GIU-DI & $4+12 / c$ & $5+10 / d$ \\
\hline GIU-A3 & $d$ & $d$ \\
\hline Giu-B3 & $g$ & $h$ \\
\hline$G l u-03$ & $c$ & a \\
\hline$G[i-A]$ & 0 & $a$ \\
\hline$G[i-B]$ & $\mathrm{g}$ & $d$ \\
\hline Gli-D! & $j$ & $a$ \\
\hline Gli-A2 & $i$ & $p$ \\
\hline$G 1 i-B 2$ & $g$ & $c$ \\
\hline$G[i-02$ & $m$ & $m$ \\
\hline
\end{tabular}

and Glu-D1, were expressed. Marius has subunits: null, $7+9$, and $4+12$ ( $c, c$, and $c$ alleles); and Cajeme 71 has subunits: $1,17+18$, $5+10(a, i$, and $d$ alleles). A total of six LMW-GS bands representing three genetic loci Glu- $A 3$, Ghu-B3 and Ghu-D3 were detected. Marius has the alleles Glu-B3g and Glu-D3c and Cajeme71 the Glu-B3h and Glu-D3a. Parental lines have the same allele for the Glu-A3 locus. A-PAGE analysis of the parental lines demonstrated that they have the same alleles for Gli-A1 and Gli-D2 loci (Table 1). Allelic variation of gliadins was limited to four loci; Marius has Gli-B1g. Gli-D1j, Gli-A2l and Gli-B2g and Cajeme71 has Gli-B1d, Gli-D1a, Gli-A2p and Gli-B2C. After analyzing all gliadins alleles, it was confirmed that gliadins encoded at the Gli-Bl and Gli-Dl loci were completely linked to the LMW glutenin subunits $G l u-B 3$ and $G l u-D 3$ respectively.

Across the two growing seasons (2005-2006 and 2006-2007). Cajeme 71 showed $1-2 \%$ more PC, $35 \%$ more $\mathrm{HI}, 60 \mathrm{~mm}$ more SDSS. 30 s longer MxT, 16\% less MTo, $60 \mathrm{~mm} \mathrm{H}_{2} \mathrm{O}$ more DTen, $70 \mathrm{~mm}$ less dough DExt, and $300\left(\mathrm{~J} \times 10^{-4}\right)$ greater DStren than Marius (Table 2). In the RILs' population, quality traits varied over a wide range and were normally distributed (Table 2). Significant differences were found between the parents and among RlLs. For most of the quality traits analyzed, positive and negative transgressive segregations were observed in some RILs, which suggest that positive and negative alleles may be found in both parental lines (Tables S1 and S2).

Correlation coefficients among the average values of quality parameters, over two seasons, are presented in Table 3. Hl has a positive correlation with DTen $(r=0.75)$ and DStren $(r=0.64)$, in agreement with previous studies (Bordes et al., 2008; Branlard et al., $2001)$. SDSS showed a positive correlation with $\mathrm{MxT}(r=0.59)$, DTen $(r=0.44)$, and DStren $(r=0.56)$, and a negative correlation with MTo $(r=-0.53)$ and DExt $(r=-0.44)$. These results confirmed those of various authors (Axford et al., 1979; Moonen et al., 1982; Preston et al., 1982). MxT exhibited a strong correlation with DTen $(r=0.78)$ and DStren $(r=0.80)$. A strong correlation $(r=0.93)$ was observed also between DTen and DStren. These results are similar to previous reports in bread wheat (Groos et al., 2004) and in durum
Table 3

Correlation coefficients among the quality parameters measured over two year's.

\begin{tabular}{|c|c|c|c|c|c|c|c|}
\hline & PC & SDSS & $M \times T$ & MTo & DTen & DExt & DStren \\
\hline $\mathrm{HI}$ & NS & NS & $0.37^{* *}$ & $0.38^{* *}$ & $0.75^{* z}$ & $0.49^{z x}$ & $0.64^{z z}$ \\
\hline PC & & NS & $0.36^{* *}$ & $0.26^{* *}$ & $0.26^{* z}$ & $0.34^{z x}$ & $0.27^{x x}$ \\
\hline 5D5S & & & $0.59^{\mathrm{k}+}$ & $0.53^{+4}$ & $0.44^{+*}$ & $0.44^{\mathrm{ee}}$ & $0.56^{\mathrm{ke}}$ \\
\hline $\mathrm{MxT}$ & & & & $0.60^{+4}$ & $0.78^{+*}$ & $0.59^{\mathrm{ke}}$ & $0.80^{\mathrm{k}}$ \\
\hline MTo & & & & & $0.64^{+*}$ & $0.45^{\circ 8}$ & $0.68^{\circ e}$ \\
\hline DTen & & & & & & $0.67^{6.8}$ & $0.93^{\circ:}$ \\
\hline DExt & & & & & & & $0.55^{x z}$ \\
\hline
\end{tabular}

${ }^{*}$ Correlation is significant at the 0.01 level. N.5: non significant.

HI: hardness index, PC: protein content, SDSS: sedimentation test, MxT: mixing time, MTo: mixing tolerance, DTen: dougl tenacity. DExt: dougl extensibility. DStren: dough strength.

wheat (Martinez et al., 2005). In the same sense, it is known that mixing requirement is related to dough strength and correlates well with bakery mixing time (Miller et al., 1956).

\subsection{QTL mapping}

Two hundred and sixty-three SSR markers out of six hundred and eighty-three (38.5\%) were found to be polymorphic in the parental lines; these markers were used for linkage analysis and mapping of the quality traits. From the SSR markers' analysis in the Marius/Cajeme71 population, 22 linkage groups and 53 unlinked markers were found with a total length of $1113.3 \mathrm{cM}$. Although this genome coverage (26\%) was low, considering that the total map size of hexaploid wheat is estimated as $4200 \mathrm{cM}$, it presented reasonable genome coverage, large enough for exploratory QTL mapping of quality traits. Given the population size 83 RILs for Marius/Cajeme71 progeny, some linkage groups showed some gaps and disagreement in the order of closely linked markers between the map produced and the consensus map within some chromosome intervals. The disagreements in marker order of closely linked markers between genetic maps and derivation of the most correct marker order can be facilitated by comparison with the published consensus map (Somers et al., 2004).

A total of 20 QTLS were detected by CIM analysis (Table 4), four out of them were consistent over two years. Their map positions are shown in Fig. 1.

\subsubsection{Sedimentation test QTLS}

One repeatable QTL from Cajeme71 was detected on chromosome 7AS, between wmc790 and gwm635 markers (QSed.upm-7AS, $R^{2}=17-24 \%$, LOD $=2.4-4.1$ ). There is no other QTL for SDSS previously reported on chromosome 7A. In the 7 homeology group has been described one QTL in chromosome 7BS (Blanco et al., 1998).

Another consistent QTL from Cajeme71 was detected on chromosome 5BS between wmc537.1 and gwm371 markers (QSed.upm$5 B S, R^{2}=17-22 \%, L O D=3.1-3.4$ ). Another QTL for SDSS has been reported on chromosome 5BS, in a population of 226 F5 RILs

Table 2

Mean, lange, standard deviation (SD) and skewness of quality traits evaluated in the parental lines and Rlls for the two years (2006-2007)

\begin{tabular}{|c|c|c|c|c|c|c|}
\hline \multirow[t]{2}{*}{ Trait } & \multicolumn{2}{|c|}{ Parental lines } & \multicolumn{4}{|l|}{ Rlls } \\
\hline & Marius & Cajeme71 & Mean & Range & 50 & 5kewness \\
\hline PTotein content (\%) & 13.87 & 15.42 & 14.57 & $12.82-16.65$ & 0.79 & 0.467 \\
\hline Hardness index & 37.80 & 70.45 & 47.66 & $19.24-90.26$ & 24.54 & 0.574 \\
\hline SDSS (mm) & 89.17 & 105.33 & 81.69 & $30.00-107.08$ & 18.87 & -0.671 \\
\hline Mixing time (s) & 45.00 & 77.25 & 67.23 & $22.00-171.00$ & 36.21 & 1.151 \\
\hline Mixing tolerance (\%) & 40.50 & 24.80 & 29.75 & $11.79-60.00$ & 7.80 & 0.118 \\
\hline Dough tenacity $\left(\mathrm{mm} \mathrm{H}_{2} \mathrm{O}\right)$ & 28.03 & 95.67 & 54.53 & $22.15-156.00$ & 35.16 & 1.462 \\
\hline Dough extensibility (mm) & 145.30 & 77.03 & 154.92 & $62.32-283.50$ & 54.15 & 0.203 \\
\hline Dough strength $\left(\mathrm{J}: 10^{-4}\right)$ & 63.16 & 377.80 & 182.93 & $60.08-466.45$ & 106.22 & 1.077 \\
\hline
\end{tabular}


Table 4

Quantitative trait loci detected in the Marius $\times$ Cajeme 71 wheat population by composite interval mapping.

\begin{tabular}{|c|c|c|c|c|c|c|c|c|}
\hline Trait & QTL & Chromosome & Position & Flanking markers & Year & $R^{2}$ & LOD & Additive effect \\
\hline \multirow[t]{5}{*}{ Sedimentation test } & QSed.upm-7AS & 7AS & 5.3 & wmc790, gwm635 & 2006 & 17 & 2.4 & -8.58 \\
\hline & & & & & 2007 & 24 & 4.1 & -10.77 \\
\hline & QSed.upm-5BS & 5BS & 16.6 & whe5 537.1 , gwm 371 & 2006 & 17 & 3.1 & 8.86 \\
\hline & & & & & 2007 & 22 & 3.4 & 10.10 \\
\hline & QSedupm-6DS & 605 & 22.5 & cfd 13.1, cfd 13.2 & 2007 & 14 & 2.5 & 7.90 \\
\hline \multirow[t]{3}{*}{ Mixing time } & OMXT.upm-3BS & 3BS & 5.5 & gwm389 & 2006 & 10 & 2.5 & 15.90 \\
\hline & OMxT.upm-1DL & $1 \mathrm{DL}$ & 23.9 & cfd92, gdm 126 & 2006 & 15 & 3.0 & -17.22 \\
\hline & & & & & 2007 & 13 & 2.8 & -18.34 \\
\hline \multirow[t]{3}{*}{ Mixing tolerance } & QMTo.upm-2AS & 2AS & 18.2 & wmc177, wme522 & 2006 & 17 & 3.3 & -7.65 \\
\hline & & & & & 2007 & 10 & 1.9 & -3.04 \\
\hline & OMTo.upm-7AS & 7AS & 0.2 & winc 633 , winc 790 & 2007 & 14 & 3.5 & 3.82 \\
\hline \multirow[t]{5}{*}{ Dougl tenacity } & QDten.upm-2AS & 2AS & 18.2 & winc177, wimc522 & 2006 & 29 & 5.6 & 19.20 \\
\hline & ODten.upm-2BS & 2BS & 4.1 & barc13.1 & 2007 & 11 & 4.8 & 12.68 \\
\hline & ODten.upm-1BS & 1BS & 0.2 & $\mathrm{dd} 48.2, G \mathrm{w}-\mathrm{B} 3$ & 2006 & 20 & 4.8 & 16.45 \\
\hline & ODten.upm-10L & 1DL & 34.7 & cfd92, gdm 126 & 2007 & 23 & 3.5 & -17.57 \\
\hline & ODtenupm-5DL & 5DL & 7.3 & gwm654 & 2006 & 14 & 2.7 & 13.50 \\
\hline \multirow[t]{4}{*}{ Dough extensibility } & ODext.upm-5AS & 5AS & 31.5 & gwm304, gwm293 & 2007 & 16 & 4.6 & -32.22 \\
\hline & ODext.upm-7AS & TAS & 3.7 & wmc 790, gwm 635 & 2006 & 17 & 3.2 & -19.66 \\
\hline & ODext.upm-2BS & 2BS & 2.5 & gwm148, bare 13.1 & 2007 & 7 & 3.1 & 19.50 \\
\hline & ODext.upm-1DL & 1DL & 36 & cfd92, gdm126 & 2007 & 29 & 6.1 & 38.78 \\
\hline \multirow[t]{4}{*}{ Dough strength } & ODstren.upm-2AS & $2 A S$ & 19.2 & wme522 & 2006 & 20 & 4.5 & 60.92 \\
\hline & ODstren.upm-1BS & 1BS & 24.3 & cfd 48.2 & 2006 & 18 & 4.6 & 58.61 \\
\hline & ODstren.upm-5DL 1 & 5DL & 24.3 & cfds & 2007 & 14 & 3.1 & -44.89 \\
\hline & ODstren.upm-5DL2 & 5DL & 72 & gwm654 & 2007 & 14 & 2.6 & -48.58 \\
\hline
\end{tabular}

${ }^{a}$ Additive effect: positive values indicate increasing effects of 'Marius' alleles, while negative values indicate increasing effects of 'Cajeme71'alleles.

derived from the cross between a winter wheat and a winter spelt (Zanetti et al., 2001) and in the homologous group 5, on chromosome 5D (Huang et al., 2006; Li et al., 2009).

The third QTL from Cajeme71 was detected on chromosome GDS between cfd13.1 and cfd13.2 (QSedupm-6DS, $R^{2}=14 \%, L O D=2.5$ ). Some other QTLs have been also reported in the homologous group 6 (Li et al., 2009; McCartney et al., 2006).

An additional QTL from Cajeme 71, close to the map positions of storage-protein Glit-D1 loci, was identified on chromosome 1D close to cfd92 at 20.9 map position in seasons 2006 and 2007. This QTL explained $12-6 \%$ of phenotypic variation with an additive effect of $7.3-5.6 \mathrm{~mm}$, but didn't reach the threshold LOD value $(L O D=2.1, L O D=1.1$, data not shown). Other authors have found QTLs in chromosome 1D with a stronger effect that also clearly corresponds to Ghu-D1 loci (Li et al., 2009; Rousset et al., 2001) or in chromosome 1B associated with Glu-B1, Glu-B2, or Glu-B3 loci (Blanco et al., 1998; McCartney et al., 2006; Patil et al., 2009).

\subsubsection{Mixograph QTLS}

3.2.2.1. Mixing development time. A repeatable QTL from Cajeme71 linked to cfd92 was identified for MxT in chromosome 1DL (QMxT. upm-1DL, $L O D=2.8-3.0 ; R^{2}=13-15 \%$ ) close to the location of the Glu-D1 locus. Several authors have reported QTLs for mixograph mixing development time close to the GIu-DI loci. For example, Campbell et al. (2001) identified a major QTL for mixograph peak time $(L O D=8.7)$ located at the Glu-Dy 1 marker in chromosome 1D. Huang et al. (2006) also reported a QTL for mixograph development time close to Glu-D1 (QMdt.crc-1D, LOD $=26.8, R^{2}=55.9 \%$ ). QTLs for other mixing time traits have also been reported close to Glu-D1 loci. Recently, Sun et al. (2008) identified a QTL for farinograph development time in chromosome 1D (QDdt.sdau-1D Glu-D1Xsrap19, $L O D=12.74, R^{2}=37.63 \%$ ) while Campbell et al. (2001) and Rousset et al. (2001) reported a QTL for mixing time. Another QTL from Marius was detected in the first season on chromosome 3BS linked to gwm389 (QMxTupm-3BS, $L O D=2.54, R^{2}=10 \%$ ). Several authors have previously reported QTLs for MxT on chromosome 3B. in studies with reciprocal substitution lines between hard wheat cultivars classified as hard or soft (Zemetra et al., 1987), and in a cross between the Canadian wheat variety 'AC Karma' and the breeding line 87E03-S2B (Huang et al., 2006). Other QTLs have been detected in homologous group 3. From a population of 101 doublehaploid lines generated from a cross between Grandin, a hard spring wheat variety, and AC Reed, a soft spring wheat variety, markers on $3 \mathrm{AS}$ that correlated with mixograph parameters were identified (Breseghello et al., 2005).

3.22.2. Mixing tolerance. One consistent QTL for MTo over two years was detected in Cajeme71 on chromosome 2AS between wmc177 and wmc522 (OMToupm-2A, LOD $=1.92-3.3$, $R^{2}=10-17 \%$ ). In the second season (2006-2007), a putative QTL for MTo inherited from Marius was detected on chromosome 7AS at map position 0.2 (QMTo.1um $-7 A S, L O D=3.5, R^{2}=14 \%$ ) between wmc633 and winc 790 .

other studies have located QTLs for MTo in other chromosomes than those identified in this study. For example, McCartney et al. (2006) identified QTLs for mixing tolerance on chromosomes 1B and 4D, Huang et al. (2006) reported the presence of QTLs on 1D and 4D, and Sun et al. (2008) identified a QTL on chromosome 1D.

\subsubsection{Alveograph QTLs}

3.2.3.1. Dough tenacity. In this study, numerous QTLs were detected for DTen: on chromosome $2 \mathrm{~A}$, inherited from the parent Marius between wmc177 and wmc522 (QDten.upm-2AS, LOD $=5.6$, $R^{2}=29 \%$ ), on chromosome 1 BS between cfd $48 \mathrm{~b} .2$ and Glu-B3 (QDten.upm-1BS, LOD $=4.8, R^{2}=20 \%$ ), on chromosome 2BS linked to barc13.1 (QDten.upm-2BS, LOD $=0.92-4.8, R^{2}=3-11 \%$ ), on chromosome 1DL close to Glu-D1 and gdm126 (QDten.upm-1DL, $L O D=3.5, R^{2}=23 \%$, and on chromosome 5DL linked to gwm654 (QDten.upm-5DL, LOD $=2.70, R^{2}=14 \%$ ). We can infer that all QTLs for alveograph parameters located on chromosome group $1 \mathrm{~B}$ close to Glu-B3 and on chromosome group $1 \mathrm{D}$ close to Glu-DI are the result of these loci.

Numerous studies have identified an association between DTen and QTLs on chromosomes 2A, 1B, 2B, 1D, 5D but also QTLs were 
$2 \mathbf{A}$

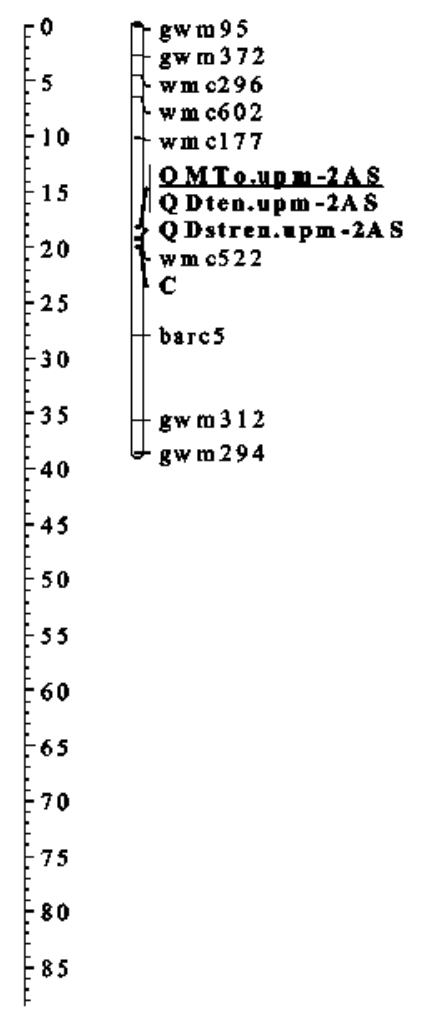

$5 \mathbf{A}$

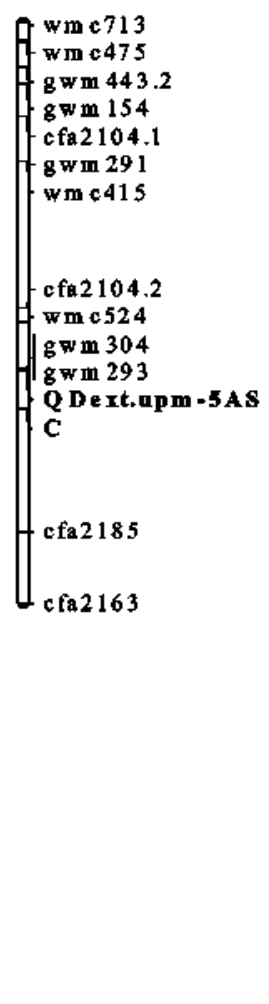

7A

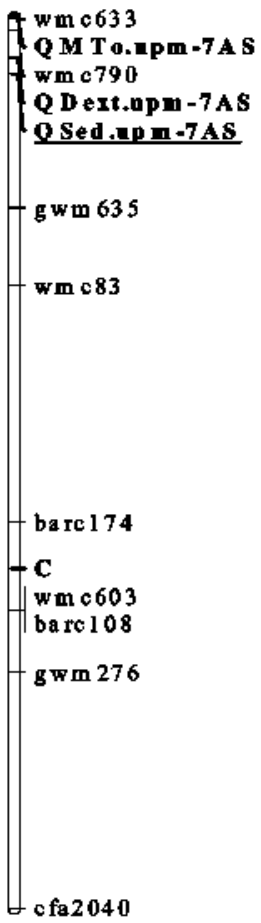

1 B

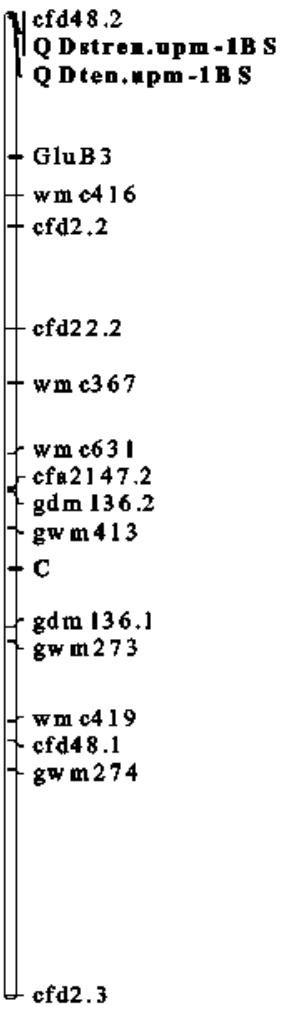

2 B

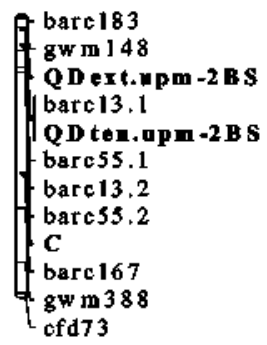

3 B 5 B

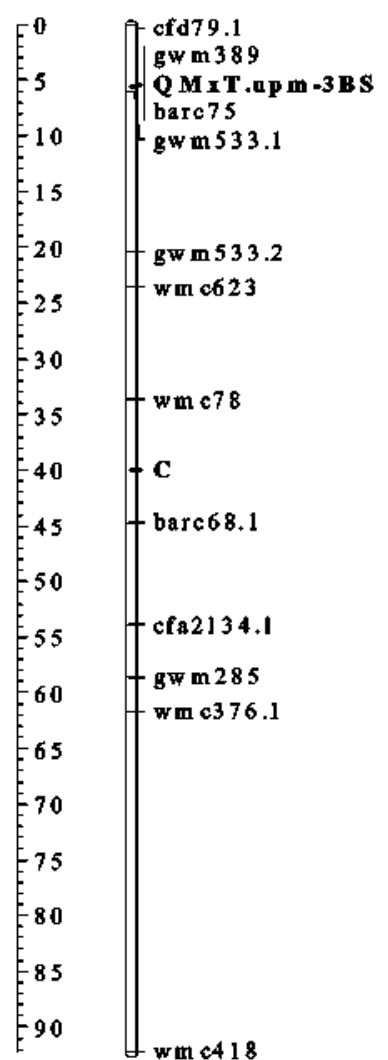

1D

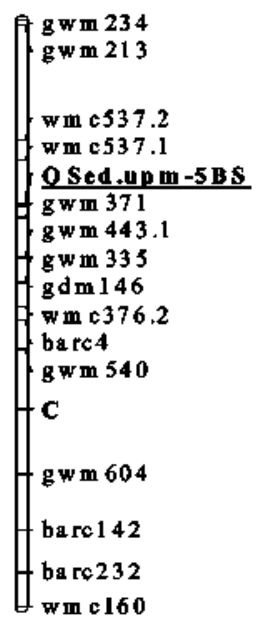

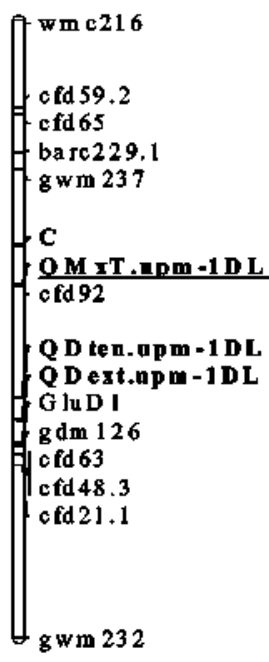

5D

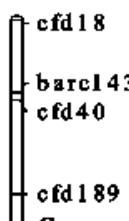

$\mathrm{C}$

gm 212

Hefd 8

O D stre n.n p m -5 D L. 1

cfd 37

cfd 29

c fd 3

$\operatorname{gmw} 16$

cfd 7.1

cfd 102

Q D ten .upm-5DL

Q D stre n.n pm -5 D L.2

gw 654
6D

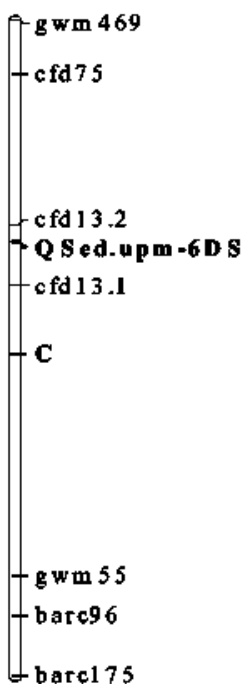

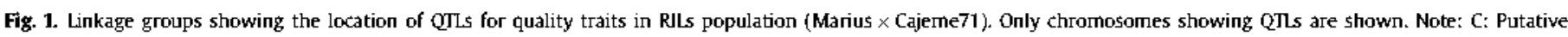
centromere. QTLs underlined are repeatable during two harvest years (2006-2007). 
detected in other chromosomes than those mentioned above. For example, Zanetti et al. (2001) identified nine QTLs for DTen that explained $48 \%$ of its variance not only on chromosomes $2 \mathrm{~A}$ and $5 \mathrm{D}$, but also, on chromosomes 4A, 4D, 5A, 5B, 7B and 7D. Another study identified twelve QTLs related to DTen on chromosomes 1B, 2A, 2B but also on 1A, 3B, 4A, 4B, 5A, 6A, 6D, 7D and one QTL linked to gwm130 (Groos et al., 2004). In addition, Nelson et al. (2006) reported that DTen was influenced by Gli-B1 alleles (from the synthetic hexaploid wheat line WPI 219) on 1BS, but also by 'Opata' alleles on 3BS. Recently, Pshenichnikova et al. (2008) identified four QTLs for DTen on chromosomes 5D, 1BL, and 4BL.

3.2.3.2. Dough extensibility. In the present work, four QTLs were detected for DExt on chromosomes: 7AS between wmc790 and gwm635 (QDextupm-7AS, LOD =3.2, $R^{2}=17 \%$ ); 5AS linked to gwm304-gwm293; (QDext.1pm-5AS, LOD $=4.6, R^{2}=17 \%$ ); $1 \mathrm{DL}$ between cfd92 and gdm126 (QDextupm-1DL, LOD $=6.1, R^{2}=29 \%$ ); and 2BS between gwm 148 and barc13.1 (QDext.upm-2BS, LOD = 3.1, $R^{2}=7 \%$ ).

Numerous studies have identified a relationship between DExt and QTLs on chromosomes 5AS, 7AS, 1DL and 2BS but other chromosomes were involved too in DExt like homeologous group 5 and 7. For example, Zanetti et al. (2001) reported four QTLs for DExt on chromosomes 2B, 3B, 5B and 7B that explained $25 \%$ of the phenotypic variance. Also, Groos et al. (2004) identified QTLs for DExt on chromosomes 2B, 5B, 1A, 1B, 3B, 4A, 4B, and 6B, and one QTL linked to gwm 130 .

Furthermore, Nelson et al. (2006) using a cross WPI $219 \times$ Opata, reported that DExt increased by WPI 219 alleles on 7AS but also by Opata alleles near Gli-A1 and Gli-B1 and by Opata alleles in the HMW Glu-A1 region on $1 \mathrm{AL}$. Lately, another QTL for dough DExt was detected on chromosome 1AL (Pshenichnikova et al., 2008).

3.2.3.3. Dough strength. A total of four QTLs were detected for DStren on chromosomes: 2AS close to wmc522 (QDstren.upm-2AS, $\mathrm{LOD}=4.5, R^{2}=20 \%$ ), $1 \mathrm{BS}$ close to Gh-B3 linked to cfd 48.2 (QDstren. upm-1BS, $L O D=4.6, R^{2}=18 \%$ ), 5DL near the centromere, linked to cfd8 (QDStren.upm-5DL.1, LOD $=3.1, R^{2}=14 \%$ ), and at the end of chromosome 5DL linked to gwm654 (QDstren.upm-5DL.2, $\mathrm{LOD}=2.6, R^{2}=14 \%$ ).

Other authors have identified QTLs for DStren on chromosomes 2AS, 1BS, and 5DL but also other chromosomes than homeologous group 1, 2 and 5 were implicated too. For example, Zanetti et al. (2001) reported QTLs for DStren not only in chromosome 1B near Gh-B3 and near centromere 2D, 5D but also on 3A, 3DL, 1AL-5AS, $5 \mathrm{~A}, 5 \mathrm{~B}$, and $7 \mathrm{~S}$ chromosomes, explaining $39 \%$ of the phenotypic variance. In addition, Groos et al. (2004) reported eleven QTLs for DStren not only on chromosomes 1B, 2D, 5A, and 5B but also on 1A. 1D, 3A, 4A, 4D, 7D, and one QTL linked to gwm130. Also, Crepieux et al. (2005) detected for dough strength, three significant QTL located on chromosomes 1A, 1B, and 1D, close to the HMW glutenin loci, in homoeologous position. Furthermore, Nelson et al. (2006) revealed that dough strength was consistently increased by alleles on $1 \mathrm{BS}$ near the Gli-B1 gliadin loci. Other QTLs were found on 2DS, 5AL and 7BL. Moreover, Arbelbide and Bernardo (2006) identified markers for dough strength, as: Xgpw1170, Xgwm264, Gh-B1, Xbarc061, and Xwmc044 on chromosome 1B; Xcfd32, GhuD1, Xgwm642, Xcfd48, Xcfd27, Xgdm126, and Xcfd63 on chromosome $1 \mathrm{DL}$ and Xgpw323 on chromosome 5D, also markers in other chromosomes like: Xgwm164, Xgwm357, Glu-A1, Xcfa2129, and Xcfa2219 on chromosome 1A; Xbarc117 on chromosome 5A; and Xgwm234 on chromosome 5B. Lately, Pshenichnikova et al. (2008) reported four QTLs for DStren on chromosome 5DL near the marker $X k s u d 30$. Two markers were found on the short and long arms of chromosome $1 \mathrm{~B}$, one on the short arm was associated with the marker Xmwg938b, located near the group of tightly linked loci Gli$B 1$ and Glu-B3 and the other QTL was localized to the long arm between markers Xcdo346b and Xcdo1189. In addition, a marker that correlated only with DStren (Xcdo1312a) was found in chromosome $4 \mathrm{~B}$. In this study, the QTLs identified for DStren on chromosome $1 \mathrm{~B}$ may be due to the influence of the glutenin locus (GluB3/Gli-B1), and the QTL QDstren.upm-5DL.1 in chromosome 5D can be influenced by the nearby presence of the Ha (Hardness) locus on this chromosome.

In conclusion, the focus of this study was to identify genome regions with dough quality effects using a set of 83 RILs. A total of twenty QTLs related to quality traits have been mapped on 10 different chromosomes. The lack of common markers between the different QTL analysis previously published, makes it difficult to assess if the QTLs detected in the different populations could be identical.

Five QTLs for mixograph and alveograph parameters, with major effects ( $L O D=2.8-6.1, R^{2}=13-29 \%$ ) have been found to be associated with Glu-B3 and Glu-D1 loci on chromosomes $1 \mathrm{~B}$ and $1 D$. These results confirm the previous investigations on the association between dough quality and storage-protein alleles (Payne, 1987). Three other QTLs were consistent over two years. The first one, located on chromosome 7A (QSed.upm-7AS), showed the highest effect ( $L O D=2.4-4.1, R^{2}=17-24 \%$ ) occurs in the same region as other QTLs detected for mixing tolerance and dough extensibility (QMTo.upm-7AS and QDext.upm-7AS). The second reliable QTL, associated with mixing tolerance, was located on chromosome 2A (QMTo.upm-2AS, LOD $=1.9-3.3, R^{2}=10-17$ ). This QTL was surrounded by others associated with alveograph parameters (QDien.upm-2AS and QDstren.upm-2AS). The presence of QTL clusters could be due to the high correlation among dough quality parameters, but also may be due to the pleiotropic effects of a single or more than one QTL. The third consistent QTL was associated with the sedimentation test (QSed.upm-5BS, LOD $=3.1-3.4, R^{2}=17-22 \%$ ) and positioned on chromosome $5 \mathrm{~B}$. No other $Q T L$ for this quality parameter was mapped before on this chromosome. These three chromosomes (7A, 2A and 5B) need to be investigated further by saturation of the genetic linkage map using a larger population.

This work could lead to the development of markers useful in the selection of segregant lines with high dough strength. This would be a complementary and efficient method of screening, especially in the early generations in which is not possible to have enough flour for running analytical dough tests as mixograph and alveograph.

In this study, some RILS showed higher dough extensibility values than the parental material and they are a very suitable population for searching QTLs associated with this trait. Although we have not found any repeatable QTL associated to extensibility. the presence of 4 putative QTLs, and the great importance of this trait in quality, require future validation studies for the development of markers for wheat breeding programs.

\section{Acknowledgements}

This work was supported by Grant (FPI) No. BES-2004-6507 and projects references AGL2003-06382 and AGL 2006-8025 from the (Spanish Ministry of Science and Technology. We gratefully acknowledge the technical staff for their excellent assistance at Escuela Técnica Superior lngenieros Agrónomos, Universidad Politécnica de Madrid, at Institut für Pflanzengenetik und Kulturpflanzenforschung (JPK), Germany, at Agriculture and Agri-Food Canada, Cereal Research Centre, Winnipeg, Canada, and at INIAP, Estaçâo Nacional de Melhoramento de Plantas, Elvas, Portugal. 


\section{Supplementary data}

Supplementary data associated with this article can be found in the online version, at doi:10.1016/j.jcs.2010.03.001.

\section{References}

American Association of Cereal Chemists, 1995. Approved Methods of the AACC. Methods 54-40A, ninth ed. The American Association of Cereal Chemists, St Paul, Minnesota. Arbelbide, M., Bernardo. R., 2006. Mixed-modelQTL mapping for kernel hardness and dough strength in bread wheat. Theoretical and Applied Genetics 112, 885-890.

Axford, D.W.E., McDermott, E.E., Redman, D.G., 1979. Note on the sodium dodecyl sulfate test of breadmaking quality: comparison with Pelshenke and Zeleny tests. Cereal Chemistry 56, 582-584.

Basten, C., Weir, B., Zeng, Z.B., 2001. OTL Cartographer. Department of Statistics, North Carolina State University, Raleigh, NC.

Blanco, A., Bellomo, M.P., Lotti, C., Maniglio, T., Pasqualone, A., Simeone, R., Troccoli, A., Di Fonzo, N., 1998. Cenetic mapping of sedimentation volume across environments using recombinant inbred lines of durum wheat. Plant Breeding 117, 413-471.

Blanco, A., Pasqualone, A., Troccoli, A., Di Fonzo, N., Simeone, R., 2002. Detection of grain protein content QTls acoss environments in tetraploid wheats. Plant Molecular Biology 48, 615-623.

Bordes, J., Branlard, G., Oury, F.X., Charmet, G., Balfourier, F., 2008. Agronomic characteristics, grain quality and flour rheology of 372 bread wheats in a worldwide core collection. Journal of Cereal Science 48, 569-579.

Branlard, G., Dardevet, M., Saccomano, R, Lagoutte, F., Gourdon, l., 2001. Genetic diversity of wheat storage proteins and bread wheat quality. Euphytica 119, 59-67.

Breseghello, F., Finney, P.l., Gaines, C., Andrews, L., Tanaka, J., Penner, G., Sorrells, M. E. 2005. Genetic loci related to kernel quality differences between a soft and a hard wheat cultivar. Crop Science 45, 1685-1695.

Bushuk, W. 1998. Wheat breeding for end-product use. Euphytica 100, 137-145.

Campbell, K.G., Finney, P.l., Bergman, C.J., Gualberto, D.G., Anderson, J.A., Giroux, M. J., 2001. Quantitative trait loci associated with milling and baking quality in a soft $\times$ hard cross. Crop 5cience 41, 1275-1285.

Cornish, G.B., Bekes, F., Allen, H.M., Martin, J.M., 2001. Flour proteins linked to quality traits in an Australian doubled haploid wheat population. Australian Journal of Agricultural Research 52, 1339-1348.

Crepieux, S., Lebreton, C, Flament, P., Charmet, G., 2005. Application of a new IBD-based mapping method to common wheat breeding population: analysis of kernel hardness and dough strength. Theoretical and Applied Genetics 111, 1409-1419.

Dick, J., Quick, J., 1983. A modified sceening test for rapid estimation of gluten strength in early-generation durum wheat breeding lines. Cereal Chemistry 60 . $315-318$.

Dobraszczyk, B.J., Morgenstern, M., 2003. Rheology and the breadmaking process. Journal of Cereal Science 38, 229-245.

Elangovan, M., Rai, R., Dholakia, B.B., lać, M.D., Tiwari, R., Gupta, R.K., Rao, V.S Röder, M.S. Gupta, V.5. 2008. Molecular genetic mapping of quantitative trait loci associated with loaf volume in hexaploid wheat (Triticum aestivum). Journal of Cereal Science 47, 587-598.

Finney. K.F., 1943. Fractionating and reconstituting techniques as tools in wheat flour research. Cereal Chemistry 20, 381-396.

Groos, C., Robert, N., Bervas, E., Charmet. G., 2003. Genetic analysis of grain protein content, grain yield and thousand-kemel weight in bread wheat. Theoretical and Applied Genetics 106, 1032-1040.

Groos, $C$., Bervas, E., Charmet, $G$., 2004. Genetic analysis of hardness and breadmaking related traits in a hard $\times$ hard bread wheat cross. Journal of Cereal 5cience 40, 93-100.

Gupta, R.B., Shepherd, K.W., 1990. Two-step one-dimensional SD5-PACE analysis of LMW subunits of glutelin. 1. Variation and genetic control of the subunits in hexaploid wheats. Theoretical and Applied Genetics 80, 65-74.

Huang, X.Q. Cloutier, S., Lycar, L., Radovanovic, N., Humphreys, D.G., Noll, J. Somers, S.D., Brown, J.P.D., 2006. Molecular detection of QTls for agronomic and quality traits in a doubled haploid population derived from two Canadian wheats (Titicum aestivum L.). Theoretical and Applied Genetics 113, 753-766.

Joppa, L.R., Du, C., Hart, G.E., Hareland, G.A., 1997. Mapping gene(s) for grain protein in tetraploid wheat (Triticum turgidum L) using a population of recombinant inbred chromosome lines. Crop Science 37, 1586-1589.

Kosambi, D.D., 1944. The estimation of map distances from recombination values. Annals of Eugenics 12, 172-175.

lafiandra, D. Kasarda, D.D. 1985, One and two-dimensional (two-pH) polyacrylamide gel electrophoresis in a single gel: separation of wheat proteins. Cereal Chemistry 62, 314-319

lander, E.S., Green, P, Abrahamson, J., Barlow, A., Daly, M.J, Lincoln, S.E., Newburg, L., 1987. Mapmaker an interactive computer package for constructing primary genetic linkage maps of experimental and natural populations. Genomics 1, 174-181.

Li, Y., Song, Y., Zhou, R., Branlard, G., Jia, J., 2009. Detection of QTls for bread-making quality in wheat using a recombinant inbred line population. Plant Breeding $128,235-243$.

Ma, W., Sutherland, M., Kammholz, S., Banks, P., Brennan, P., Bovill, W., Daggard, G., 2007. Wheat flour protein content and water absorption analysis in a doubled haploid population. Joumal of Cereal Science 45, 302-308.
MacRitchie, F., 1994. Role of polymeric proteins in flour functionality. In: S. Martino al Cimino (Ed.). Wheat Kernel Proteins: Molecular and Functional Aspects. Universita Degli Studi Della Tuscia, Consiglio Nazionale della Richerche, Viterbo, Italy. pp. 145-150.

Mann, G., Diffey, 5., Cullis, B., Azanza, F., Martin, D., Kelly, A., Mclntyre, l. Schmidt, A., Ma, W. Nath, Z., Kutty, l., Leyne, P.E., Rampling, L, Ouail, K.l. Morell, M.K., 2009. Genetic control of wheat quality: interactions between chromosomal regions determining protein content and composition, dough rheology, and sponge and dough baking properties. Theoretical and Applied Genetics 118, 1519-1537.

Martinez, M.C., Ruiz, M., Carrillo, J.M., 2005. Effects of different prolamin alleles on durum wheat quality properties. Journal of Cereal Science 41, 123-131.

McCartney, CA., Somers, D.]., Lukow, 0., Ames, N., Noll, l., Cloutier, S., Humphreys, D. G. McCallum, B.D., 2006. QTL analysis of quality traits in the spring wheat cross RL4452 $\times$ AC Domain. Plant Breeding 125, 565-575.

Metakovsky, E.V., Gómez, M. Vázquez, J.F., Carrillo, J.M., 2000. Hiğh genetic divessity of Spanish common wheats as judged from gliadin alleles. Plant Breeding $119,37-42$.

Miller, B.S., Hays, B., Johnson, J.A., 1956. Correlation of farinograph, mixograph, sedimentation and baking data for hard red winter wheat flour samples varying widely in quality. Cereal Chemistry 33, 277-290.

Moonen, J.H.E., Scheepstra. A., Graveland, A., 1982. Use of 5D5-sedimentation test and SDS-polyacrylamide gel electrophoresis for screening breeder's' samples of wheat for breadmaking quality. Euphytica 31, 677-690.

Nelson. J.C., Andreescu. C., Breseghello. F., 2006. Quantitative trait locus analysis of wheat quality traits. Euphytica 149, 145-159.

Patil, R.M., Oak, M.D., Tamlıankar, 5.A., Rao, V.S., 2009, Molecular mapping of QTLs for gluten strength as measured by sedimentation volume and mixograph in durm wheat (Triticum turgidum L. ssp durum). Journal of Cereal Science 49 $378-386$.

Payne, P.., 1987. Genetics of wheat storage proteins and the effect of allelic variation on bread making quality. Annual Review of Plant Physiology 38. $141-153$.

Payne, P.]., Nightingale, M.A., Krattiger, A.F., Holt, L.M., 1987. The relationship between HMW glutenim subunit composition and the bread making quality of British grown wheat varieties. Journal of the Science of Food and Agriculture 40 51-65.

Payne, P.l. Corfield, K.G., 1979. Subunit composition of wheat glutenin proteins, isolated by gel filtration in a dissociating medium. Planta 145, 83-88.

Perretant, M.R., Cadalen, T., Cliarmet, G., Sourdille, P., Nicolas, P., Boeuf, C., Tixier, M. H., Branlard, G., Bernard, S., Bernard, M., 2000. OTL analysis of bread-making quality in wheat using a doubled haploid population. Theoretical and Applied Genetics 100, 1167-1175.

Pomeranz, Y, 1988. Composition and functionality of wheat flour components. In: Pomeranz, Y. (Ed.). Wheat: Chemistiy and Technology. American Association of Cereal Chemists, St. Paul, USA, pp. 219-370.

Preston, K.R., Marcl, P.R., Tipples, K.H., 1982. An assessment of the sedimentation test of the prediction of Canadian bread wheat quality. Canadian Journal of Plant Science 62, 545-553.

Psheniclunikova, T.A. Ermakova, M.F., Chistyakova, A.K., Shchukina, I.V, Berezovskaya, E.V., Lochwasser, U., Röder, M., Börner, A., 2008. Mapping of the quantitative trait loci (QTL) associated with grain quality characteristics of the bread wheat grown under different environmental conditions. Russian Journal of Genetics 44, 74-84.

Rousset, M., Brabant, P., Kota, R.S., Dubcovsky. J., Dvorak, J., 2001. Use of recombinant substitution lines for gene mapping and QTL analysis of bread making quality in wheat. Euphytica 119, 81-87.

5omers, D.J., Isaac, P., Edwards, K, 2004. A high-density microsatellite consensus map for bread wheat (Titicam aestinam L.). Theoretical and Applied Genetics 109. 1105-1114.

SAS Institute Inc., 1996. The SAS System for Windows, 6.12 ed. SAS Institute Inc. Cary, N.C., U.S.A.

Saxton, A.M. 1998. A macro for converting mean separation output to letter groupings in Proc Mixed. In: Proc. 23rd SAS Users Group Int. 22-25 March Nashville. TN 5AS Inst. Cary. NC, pp. 1243-1246. Available at. littp:ff animalscience.ag.utk.edu/faculty/saxton/software.htm

Sourdille, P., Cadalen, T., Guyomarc'h, H., Snape, J.W., Perretant, M.R., Charmet, G. Boeuf, C., Bernard, 5., Bernard, M., 2003. An update of the Courtot $\times$ Chinese Spring intervarietal molecular marker linkage map for the OTL detection of agronomic traits in wheat. Theoretical and Applied Genetics 106, 530-538.

Sun, H., Jianhua, L., Yuding. F., Yan, Z., Fanmei, K., Rijun, l., Honggang. W., Sishen, l. 2008. Quantitative trait loci (QTLs) for quality traits related to protein and starch in wheat. Progress in Natural Science 18, 825-831.

Suprayogui, Y., Pozniak, C.J., Clarke, F.R., Clarke, J.M., Knox, R.E., Singh, A.K., 2009. ddentification and validation of quantitative trait loci for grain protein concentration in adapted Canadian durum wheat populations. Theoretical and Applied Genetics $119,437-448$

Turner, A.5., Bradburne, R.P., Fish, l., Snape. J.W. 2004. New quantitative trait loci influencing grain texture and protein content in bread wheat. Journal of Cereal Science $40,51-60$

Zanetti, S., Winzeler, M., Feuillet, C., Keller, B., Messmer, M., 2001. Genetic analysis of bread-making quality in wheat and spelt. Plant Breeding 120, 13-19.

Zemetra, R.5., Morris, R., Mattern, P.J., Sejp. L., 1987. Gene locations for flour quality in winter wheat using reciprocal chromosome substitutions. Crop Science 27, $677-681$ 